\title{
Myths and Truths about Placebo Effect in Rehabilitation for Musculoskeletal Pain
}

\author{
Giovanni Iolascon · Antimo Moretti
}

Received: July 2, 2021 / Accepted: August 12, 2021 / Published online: September 2, 2021

(C) The Author(s) 2021

\section{ABSTRACT}

This commentary provides the authors' point of view about the biopsychosocial perspective of placebo effect on musculoskeletal pain in the rehabilitation field.

Keywords: Rehabilitation; Physical therapy; Placebo; Placebo effect; Pain; Musculoskeletal pain

\section{Key Summary Points}

Rehabilitation interventions, including physical therapies, play a leading role in the management of musculoskeletal pain, though their effects should still be clarified.

It is often argued that the effects of rehabilitation interventions are due to the "placebo effect". This commentary provides the biopsychosocial perspective of placebo effect on musculoskeletal pain in rehabilitation.

G. Iolascon $(\varangle) \cdot$ A. Moretti

Department of Medical and Surgical Specialties and Dentistry, University of Campania Luigi Vanvitelli, Via de Crecchio, 6, 80138 Naples, Italy

e-mail: giovanni.iolascon@unicampania.it
In randomized controlled trials performed in a rehabilitation setting, for patients included in the placebo group (untreated or receiving sham physical therapy), there are beneficial physiological and psychological reactions due to the personality of the patient, the relationship between the therapist and the patient, the type of environment and location where rehabilitation is provided, the use of complicated devices or equipment, and the patient's expectations of improvement and/or recovery. The neurophysiological basis of a placebo effect in rehabilitation concerns psychoneuro-endocrine-immunology.

Psychological mechanisms can induce a therapeutic effect in chronic pain conditions through the conscious anticipation of the benefit induced by the therapy, the conscious conditioning due to the sight of sophisticated instruments, and the unconditioned stimulus due to the administration of therapy. 
Placebo analgesia results from different pain modulation mechanisms: the activation of descending circuits involving the dorsolateral prefrontal cortex, rostral anterior cingulate cortex, and periaqueductal gray; the modulation of pain processing by inhibiting the middle and posterior cingulate cortex, insula and thalamus; the inhibition of cholecystokinin (CCK); the activation of endocannabinoid system; and the modulation of prostaglandins synthesis.

\section{COMMENTARY}

Rehabilitation is a complex process that uses different methods in terms of mechanisms of action and therapeutic efficacy; among these, physical therapy (PT) plays a leading role but is probably also the one that most perplexes those who try to give a solid scientific consistency to anecdotal therapeutic experiences. There is no doubt that PTs, such as therapeutic exercise and physical agents (e.g., TENS, laser therapy, magnetotherapy), improve persistent musculoskeletal pain in most of the patients treated, but still today we are not able to clarify the mechanisms by which these treatments often lead to symptomatic and functional improvements of the medical condition for which they were prescribed $[1,2]$.

It is very often argued that the effects of these interventions are due to the "placebo effect". The word placebo appears for the first time in a medical scientific document in Motherby's New Medical Dictionary (1785), in which it is identified as a common medical practice [3], unlike what was described 50 years later by the Oxford Medical Dictionary, which defines it as a "Therapeutic activity attributable solely to the imagination, essentially a therapeutic illusion".

The placebo effect is evoked when a fictitious therapeutic intervention causes an improvement in a patient's condition due to mental mechanisms associated with the therapeutic act [4].

A placebo is commonly used in the family management of non-severe illnesses (e.g., the sugar pill), or sometimes even in a hospital setting (isotonic saline solution) [5]. On the other hand, the placebo effect is also recognized in part in complex treatments, including some mini-invasive techniques (intra-articular injection) or surgical techniques [6].

Normally, the concept of placebo is associated with an inert substance which is administered to the patient for the psychological effects it may have, or for research purposes in randomized controlled trials (RCTs) that test new pharmacological substances where the placebo is given to the control group [7].

In rehabilitation, the control group usually consists of patients who are not treated with "usual care" or are not treated at all. Surprisingly, even in untreated subjects, as well as in those who receive inert substances in RCTs, significant improvements of the clinical condition being treated are often reported. In these individuals, physiological and psychological reactions occur which are associated with different factors, such as the personality of the patient, the relationship between the therapist (both doctor and physical therapist) and the patient, the setting of the rehabilitation treatment, including the type of environment and location, the patient's expectations of improvement and/or recovery, and familiarity/ ecology of the rehabilitation method/technique adopted [8]. Just as in drug therapy, where a non-secondary role of the "psychological" component in providing the desired effect is reported, even more so in rehabilitation medicine, there may be unexpected effects of interventions administered, for example, on a manual mechanical approach.

Pharmacological studies have shown that the administration of an inert substance can activate the endogenous and opioid system and the endocannabinoid system [9]. For example, in the opioid system, conditioning protocols that used opioid drugs like morphine to activate a placebo analgesia proved that a $\mu$-opioid antagonist like naloxone produced a block of the effect of placebo analgesia. Similarly, in the 
cannabinoid system, drugs like ketorolac that activate the placebo analgesia were blocked by a CB1-antagonist, for example, rimonabant. In the study of pro-nociceptive cholecystokinin (CCK), it has been shown that CCK antagonists (i.e., proglumide) increase the placebo analgesia while CCK-2 agonists (i.e., pentagastrin) block it. An important discovery is that placebos and nocebos modulate the synthesis of prostaglandins which are the focus of anti-inflammatory drugs.

In summary, these studies show that both inert substances and drugs can have similar biochemical effects. It has been observed that, when patients were informed of possible side effects of a treatment, their negative expectations contributed to significantly increasing the frequency of the adverse effects. For example, in a study in which patients with benign prostatic hypertrophy were treated with finasteride, when they were informed of erectile dysfunction (ED) as a possible side effect, this was reported 3 times more often than when it was not mentioned [10]. In another study, patients treated with the $\beta$-blocker atenolol, exhibited ED only in $3.1 \%$ of the cases when they were unaware of the name of the drug, $15.6 \%$ when they knew the name but not the potential side effects, and $31.2 \%$ when they were told both the name and the possible ED, which shows a significant increase while the same drug was being used but the patients were differently informed [11]. These studies demonstrate how, for some side effects, the patient's knowledge of potential adverse events can influence and remarkably increase the chances of these events happening.

Research in recent years has sought to better understand the neurophysiological basis of this phenomenon within a new discipline, which brings together neurology, endocrinology, immunology, and psychology under the decidedly complex term of psycho-neuro-endocrineimmunology [12]. It has, however, now been established that within the placebo effect are included symptomatic and functional improvements due to various factors, such as the possibly spontaneous remission of the symptoms characterizing the pathological process (i.e., natural history); the phenomenon of regression for which, if a symptom is close to its maximum intensity during the first evaluation, it will presumably be of lesser intensity at the subsequent evaluation; the operator-dependent variability in detection of the symptoms; and undeclared factors, such as dietary or lifestyle changes that can improve the clinical picture [13]. All these factors can therefore alter the outcome when evaluating the efficacy of the placebo in a clinical trial. Another interesting issue which may cause confusion in the evaluation of efficacy is the "Hawthorne effect", which refers to a behavioral change that occurs in an individual when he is aware that he is being observed, as occurs during experimental research. This phenomenon was discovered during research on the possible relationship between the work environment and the productivity of workers at the Hawthorne plant of Western Electric. The typists and workers who were the object of the researchers' attention produced more because they were chosen from the others [14]. Similarly, the subjects of a trial show improvements simply because they have been recruited and are the object of the attention of the researchers. An important factor that plays a leading role in the genesis of a "placebo effect" during rehabilitation therapy (manual or instrumental) is the psychosocial context, in which both the patient and the rehabilitative process are placed, and includes the location where rehabilitation takes place (hospital, outpatient, home), the attitude and approach of the therapist, the use of sophisticated devices or equipment, and so on [15]. Psychological mechanisms that can induce a therapeutic effect are also to be considered, especially in complex and challenging conditions, such as chronic pain. In this context, the conscious anticipation consists of the patient's positive expectation and consequent anticipation of the benefit induced by the therapy, while in the conscious conditioning, simply the sight of sophisticated instruments induces a conditioned stimulus that, associated with the unconditioned stimulus of the actual administration of therapy, can induce a clinical improvement.

If the psychosocial context of the rehabilitation treatment could play a role in the genesis of the placebo effect, particularly in chronic 
pain syndromes, a context perceived as negative could give rise to negative expectations and results ("nocebo effects"). In fact, negative expectations can bring about a rise in pain and make analgesic drugs less effective. This is referred as nocebo hyperalgesia, which has been shown through brain imaging techniques and can last for many months [16]. For example, if the patient is informed of an initial increase in pain during the administration of rehabilitation treatment, this may induce hyperalgesia, which is modulated by CCK, with consequent increase in CCK-dependent anxiety [9]. The planning of rehabilitation interventions should consider the possible roles of placebo and nocebo mechanisms, considering that the therapeutic outcome of a rehabilitation process depends on the combination of different variables (e.g., real efficacy of the treatment, contextual factors, placebo effect). It would be extremely useful to accurately investigate these factors in the rehabilitation context, for example, by designing RCTs in which patients treated with the same rehabilitation method are compared in different contexts and for which a different impact of the placebo effect is therefore assumed.

Concerning the biology of placebo, neuroimaging studies have shown that placebo analgesia and opioid analgesia share a common neural mechanism. A placebo activates a descending pain modulation circuit that uses endogenous opioids as neuromodulators which involves activation of the dorsolateral prefrontal cortex, the rostral anterior cingulate cortex, and the periaqueductal gray. Furthermore, placebo administration inhibits the middle and posterior cingulate cortex, the insula and the thalamus, which are involved in pain processing [17]. It should be noted that the opioid systems activated with a placebo affect not only pain modulation but also respiration, and autonomic nervous system functions.

The inhibition of the anti-opioid activity of CCK further improves placebo-induced analgesia [18]. Moreover, non-opioid-mediated mechanisms modulate the placebo effect, such as those involved in hormonal control [19], while the non-secondary role of endocannabinoids in placebo-induced analgesia has also been hypothesized [20]. Finally, genetic variants, defined as "placebome", seem to affect the placebo effect, particularly gene polymorphisms of endogenous opioids, dopamine, and endocannabinoids in the central nervous system, suggesting a genetic predisposition to enhanced placebo responses [19].

There are few studies on the placebo effect on physical therapies (Table 1), but there are some RCTs which have compared PTs versus placebo. For example, one study evaluated TENS versus placebo analgesia in young and the elderly. Unlike the young, old people showed a significant pain decrease after placebo treatment $(15 \%$ vs. $40 \%$ pain reduction) $(p<0.05)$ [21]. Another study compared extracorporeal shock wave therapy (ESWT) and placebo in patients affected by plantar fasciitis [22]. Pain reduction was observed in both groups $(p<0.001)$, even though mostly in the ESWT group $(p=0.049)$. For laser therapy, a recent study showed that, in patients with osteoarthritis, placebo therapy had results comparable to laser therapy in knee pain reduction, and in improving knee range of motion [23]. Unlike laser therapy, different studies have shown that magnetotherapy is better than placebo in improving pain, stiffness, and physical function in patients with osteoarthritis [24, 25].

In the future, the rehabilitation scientific community can evaluate the influence of physical therapy treatments with greater accuracy if it has a greater understanding of contextual factors, and of placebo and nocebo effects. How patient outcomes are impacted by contextual factors should be studied. The creation of a proper trial has not been very successful among scholars [26]. A research agenda should be followed by primary studies which aim to evaluate the effect of contextual factors on many patients' clinical outcomes and in diverse health conditions. It is necessary to use RCTs to juxtapose the same PT treatment, executed in a neutral or enriched context, to measure the variation of subjective results, such as disability, pain, expectation, and satisfaction versus objective results, such as salivary cortisol, heart rate variability, and electromyographic activity. How patients perceive contextual factors should be examined by researchers. Various 
Table 1 Examples of placebo effect in rehabilitation

\begin{tabular}{ll}
\hline $\begin{array}{l}\text { Rehabilitation } \\
\text { technique }\end{array}$ & Placebo effect \\
\hline Exercise & $\begin{array}{c}\text { Pain can be reduced by both isotonic exercise and the reinforcement of participants' } \\
\text { expectations [30] }\end{array}$ \\
$\begin{array}{l}\text { Manual therapy } \\
\text { Facilitatory kinesio tape } \\
\text { (FKT) }\end{array}$ & $\begin{array}{l}\text { FKT seems ineffective in promoting muscle activity when individuals are not influenced by } \\
\text { placebo response [32] }\end{array}$ \\
TENS & $\begin{array}{c}\text { Active TENS results in 47\% pain-relief versus } 42 \% \text { of the sham TENS group in patients with } \\
\text { low back pain [33] }\end{array}$ \\
\hline
\end{tabular}

surveys and interviews have investigated how a patient perceives intervention with a placebo [27], but only one study has considered the influence of the patient's point of view by studying contextual factors in patients with musculoskeletal pain [28]. To evaluate the patient's perception of the healthcare experience in its totality a list of various contextual factors has been presented [29]. Patients can, consequently, by answering a questionnaire, be classified by their preferred contextual factors. In conclusion, the challenge for the future is to enrich physiotherapy treatment with contextual elements to establish the impact and influence of these elements on the rehabilitation outcomes to be achieved.

This article is based on previously conducted studies and does not contain any new studies with human participants or animals performed by any of the authors.

\section{ACKNOWLEDGEMENTS}

Funding. No funding or sponsorship was received for this study or publication of this article.

Authorship. All named authors meet the International Committee of Medical Journal Editors (ICMJE) criteria for authorship for this article, take responsibility for the integrity of the work as a whole, and have given their approval for this version to be published.

Authorship Contributions. Conceptualization, G.I., A.M.; writing-original draft preparation, G.I., A.M.; writing-review and editing, G.I., A.M.; both authors have read and agreed to the published version of the manuscript.

Disclosures. Giovanni Iolascon and Antimo Moretti have nothing to disclose.

Compliance with Ethics Guidelines. This article is based on previously conducted studies and does not contain any new studies with human participants or animals performed by any of the authors.

Data Availability. Data sharing is not applicable to this article as no datasets were generated or analyzed during the current study.

Open Access. This article is licensed under a Creative Commons Attribution-NonCommercial 4.0 International License, which permits any non-commercial use, sharing, adaptation, distribution and reproduction in any medium or format, as long as you give appropriate credit to the original author(s) and the source, provide a link to the Creative Commons licence, and indicate if changes were made. The images or other third party material in this article are included in the article's Creative Commons licence, unless indicated otherwise in a credit line to the material. If material is not included 
in the article's Creative Commons licence and your intended use is not permitted by statutory regulation or exceeds the permitted use, you will need to obtain permission directly from the copyright holder. To view a copy of this licence, visit http://creativecommons.org/licenses/bync/4.0/.

\section{REFERENCES}

1. Rakel B, Barr JO. Physical modalities in chronic pain management. Nurs Clin N Am. 2003;38:477-94.

2. Moretti A, Gimigliano F, Paoletta M, Liguori S, Toro G, Aulicino M, Conversano S, Iolascon G. Efficacy and effectiveness of physical agent modalities in complex regional pain syndrome type I: a scoping review. Appl Sci. 2021;11:1857. https://doi.org/10. 3390/app11041857.

3. Finniss DG. Placebo effects: historical and modern evaluation. Int Rev Neurobiol. 2018;139:1-27. https://doi.org/10.1016/bs.irn.2018.07.010.

4. Finniss DG, Kaptchuk TJ, Miller F, Benedetti F. Biological, clinical, and ethical advances of placebo effects. Lancet. 2010;375(9715):686-95. https://doi. org/10.1016/S0140-6736(09)61706-2.

5. Munnangi S, Sundjaja JH, Singh K, Dua A, Angus LD. Placebo effect. StatPearls [Internet]. Treasure Island (FL): StatPearls Publishing; 2021 PMID: 30020668 .

6. Yu SP, Ferreira ML, van Middelkoop M, Bierma-Zeinstra SMA, Zhang W, Deveza LA, Hunter DJ. Predictors of placebo response to local (intra-articular) therapy in osteoarthritis: an individual patient data meta-analysis protocol. BMJ Open. 2019;9(5): e027372. https://doi.org/10.1136/bmjopen-2018027372 .

7. Vase L, Amanzio M, Price DD. Nocebo vs. placebo: the challenges of trial design in analgesia research. Clin Pharmacol Ther. 2015;97(2):143-50. https:// doi.org/10.1002/cpt.31.

8. Testa M, Rossettini G. Enhance placebo, avoid nocebo: How contextual factors affect physiotherapy outcomes. Man Ther. 2016;24:65-74. https:// doi.org/10.1016/j.math.2016.04.006.

9. Rossettini G, Camerone EM, Carlino E, Benedetti F, Testa M. Context matters: The psychoneurobiological determinants of placebo, nocebo and context-related effects in physiotherapy. Arch
Physiother. 2020;10:11. https://doi.org/10.1186/ s40945-020-00082-y.

10. Mondaini N, Gontero P, Giubilei G, Lombardi G, Cai T, Gavazzi A, et al. Finasteride $5 \mathrm{mg}$ and sexual side effects: how many of these are related to a nocebo phenomenon? J Sex Med. 2007;4(6): 1708-12. https://doi.org/10.1111/j.1743-6109. 2007.00563.x.

11. Silvestri A, Galetta P, Cerquetani E, Marazzi G, Patrizi R, Fini M, et al. Report of erectile dysfunction after therapy with beta-blockers is related to patient knowledge of side effects and is reversed by placebo. Eur Heart J. 2003;24(21):1928-32. https:// doi.org/10.1016/j.ehj.2003.08.016.

12. França K, Lotti TM. Psycho-neuro-endocrine-immunology: a psychobiological concept. Adv Exp Med Biol. 2017;996:123-34. https://doi.org/10. 1007/978-3-319-56017-5_11.

13. Pollo A, Carlino E, Benedetti F. Placebo mechanisms across different conditions: from the clinical setting to physical performance. Philos Trans R Soc Lond B. 2011;366(1572):1790-8. https://doi.org/10. 1098/rstb.2010.0381.

14. Sedgwick P, Greenwood N. Understanding the Hawthorne effect. BMJ. 2015;4(351): h4672. https://doi.org/10.1136/bmj.h4672.

15. Peiris N, Blasini M, Wright T, Colloca L. The placebo phenomenon: a narrow focus on psychological models. Perspect Biol Med. 2018;61(3):388-400. https://doi.org/10.1353/pbm.2018.0051.

16. Meissner $\mathrm{K}$, Kohls N, Colloca L. Introduction to placebo effects in medicine: mechanisms and clinical implications. Philos Trans $\mathrm{R}$ Soc Lond B. 2011;366(1572):1783-9. https://doi.org/10.1098/ rstb.2010.0414.

17. Bingel $\mathrm{U}$, Wanigasekera $\mathrm{V}$, Wiech $\mathrm{K}$, Ni Mhuircheartaigh R, Lee MC, Ploner M, Tracey I. The effect of treatment expectation on drug efficacy: imaging the analgesic benefit of the opioid remifentanil. Sci Transl Med. 2011;3(70):70. https://doi.org/10.1126/ scitranslmed.3001244.

18. Benedetti F, Amanzio M, Vighetti S, Asteggiano G. The biochemical and neuroendocrine bases of the hyperalgesic nocebo effect. J Neurosci. 2006;26(46): 12014-22. https://doi.org/10.1523/JNEUROSCI. 2947-06.2006.

19. Amanzio M, Benedetti F. Neuropharmacological dissection of placebo analgesia: expectation-activated opioid systems versus conditioning-activated specific subsystems. J Neurosci. 1999;19(1):484-94. https://doi.org/10.1523/JNEUROSCI.19-01-00484. 1999. 
20. Theodosis-Nobelos P, Filotheidou A, Triantis C. The placebo phenomenon and the underlying mechanisms. Hormones (Athens). 2021;20(1):61-71. https://doi.org/10.1007/s42000-020-00243-5.

21. Daguet I, Bergeron-Vézina K, Harvey MP, Martel M, Léonard G. Transcutaneous electrical nerve stimulation and placebo analgesia: is the effect the same for young and older individuals? Clin Interv Aging. 2018;13:335-42. https://doi.org/10.2147/CIA. S152906.

22. Vahdatpour B, Sajadieh S, Bateni V, Karami M, Sajjadieh H. Extracorporeal shock wave therapy in patients with plantar fasciitis. A randomized, placebo-controlled trial with ultrasonographic and subjective outcome assessments. J Res Med Sci. 2012;17(9):834-8.

23. Nasirzade A, Sadeghi H, Mokhtarinia HR, Rahimi A. A review of selected factors affecting gait symmetry. Phys Treatm. 2017;7(1):3-12. https://doi.org/10. 29252/NRIP.PTJ.7.1.3.

24. Yang X, He H, Ye W, Perry TA, He C. Effects of pulsed electromagnetic field therapy on pain, stiffness, physical function, and quality of life in patients with osteoarthritis: a systematic review and meta-analysis of randomized placebo-controlled trials. Phys Ther. 2020;100(7):1118-31. https://doi. org/10.1093/ptj/pzaa054.

25. Wuschech $H$, von Hehn U, Mikus E, Funk RH. Effects of PEMF on patients with osteoarthritis: results of a prospective, placebo-controlled, doubleblind study. Bioelectromagnetics. 2015;36(8): 576-85. https://doi.org/10.1002/bem.21942.

26. Carlino E, Vase L. Can knowledge of placebo and nocebo mechanisms help improve randomized clinical trials? Int Rev Neurobiol. 2018;138:329-57. https://doi.org/10.1016/bs.irn.2018.01.012.
27. Linde $\mathrm{K}$, Atmann O, Meissner K, et al. How often do general practitioners use placebos and non-specific interventions? Systematic review and meta-analysis of surveys. PLoS ONE. 2018;13(8):e0202211. https://doi.org/10.1371/journal.pone.0202211.

28. Rossettini G, Palese A, Geri T, Mirandola M, Tortella $\mathrm{F}$, Testa M. The knowledge of contextual factors as triggers of placebo and nocebo effects in patients with musculoskeletal pain: findings from a national survey. Front Psychiatry. 2019;10:478. https://doi. org/10.3389/fpsyt.2019.00478.

29. Greco CM, Yu L, Johnston KL, et al. Measuring nonspecific factors in treatment: item banks that assess the healthcare experience and attitudes from the patient's perspective. Qual Life Res. 2016;25(7): 1625-34. https://doi.org/10.1007/s11136-0151178-1.

30. Colloca L, Corsi N, Fiorio M. The interplay of exercise, placebo and nocebo effects on experimental pain. Sci Rep. 2018;8(1):14758. https://doi. org/10.1038/s41598-018-32974-2.

31. Bialosky JE, Bishop MD, Penza CW. Placebo mechanisms of manual therapy: a sheep in wolf's clothing? J Orthop Sports Phys Ther. 2017;47(5):301-4. https://doi.org/10.2519/jospt.2017.0604.

32. Mak DN, Au IP, Chan M, Chan ZY, An WW, Zhang JH, Draper D, Cheung RT. Placebo effect of facilitatory Kinesio tape on muscle activity and muscle strength. Physiother Theory Pract. 2019;35(2): 157-62. https://doi.org/10.1080/09593985.2018. 1441936.

33. Deyo RA, Walsh NE, Martin DC, Schoenfeld LS, Ramamurthy S. A controlled trial of transcutaneous electrical nerve stimulation (TENS) and exercise for chronic low back pain. N Engl J Med. 1990;322: 1627-34. 